ARTÍCULO ORIGINAL

Recibido:

17/03/2018

Aprobado:

31/10/2019

\title{
Problemáticas en la función docente desde la perspectiva del profesorado novel universitario
}

Problems in the teaching function from the perspective of the university novel teacher

Guerrero Gallardo Héctor Iván
https://orcid.org/0000-0002-3026-3720
Universidad Central del Ecuador
Email: higuerrero@uce.edu.ec

\section{RESUMEN}

Actualmente, la indagación de las dificultades que atraviesa el profesorado novel universitario es imprescindible para la generación de alternativas que permitan apoyar a su desarrollo profesional. El objetivo propuesto, logró establecer las problemáticas del profesorado novel universitario en su función docente. Este estudio, responde a un enfoque cualitativo. Los sujetos investigados, fueron 68 docentes nuevos de la Universidad Central del Ecuador. En la metodología, se empleó la entrevista mediante una guía de base estructurada con la finalidad de comprender el significado de las narrativas de los participantes. Los resultados obtenidos, permitieron develar que; del total de protagonistas, se demostró que un 34,10\% de profesores noveles, señaló que sus problemáticas en la función docente, responden a la falta de conocimientos básicos de los estudiantes respecto a las asignaturas que imparten. Mientras un 56,65\% de participantes, afirmó que sus limitaciones responden a condicionantes institucionales. Finalmente, un 9,25\% de noveles, mencionó que sus inconvenientes, se deben a la poca experiencia que tienen en asignaturas nuevas, las cuales son asignadas en sus unidades académicas. Se concluyó, que los problemas del profesorado novel universitario en sus funciones docentes, son: la falta de apoyo de las autoridades para atender sus requerimientos a nivel de aula, bajos niveles de dominio de conocimiento de los estudiantes y la poca experiencia profesional del profesorado en asignaturas nuevas que les son establecidas conforme necesidades de las carreras profesionales.

\section{Palabras clave:}

Problemas del profesorado novel; desarrollo profesional universitario.

\footnotetext{
ABSTRACT

Currently, the investigation of the difficulties that the new university faculty is going through is essential for the generation of alternatives that allow to support their professional development.
}

The proposed objective allowed to establish the problems of the new university teaching staff in their teaching function. This study responds to a qualitative approach. The subjects investigated were 68 new teachers from the Central University of Ecuador. In the methodology, the interview was used through a structured base guide in order to understand the meaning of the narratives of the participants. The results achieved, allowed to reveal that, of the total of protagonists, it was demonstrated that a $34.10 \%$ of novice teachers, pointed out that their problems in the teaching function, respond to the lack of basic knowledge of the students regarding the subjects taught. While $56.65 \%$ of participants said that their limitations respond to institutional constraints. Finally, a $9.25 \%$ of beginners, mentioned that their drawbacks, are due to the little experience they have in new subjects, which are assigned in their academic units. It was concluded that the problems of the new university teaching staff in their teaching functions are: the lack of support from the authorities to meet their requirements at the classroom level, low levels of knowledge mastery of the students and the little professional experience of the teaching staff. new subjects that are established according to the needs of professional careers.

\section{Keywords:}

Novel teacher problems; university professional development.

\section{INTRODUCCIÓN}

El nuevo milenio se está caracterizando, por los constantes cambios que se están originando en el mundo en cuanto a sociedad, política, educación, economía, tecnología y trabajo. Por ende, las instituciones educativas de nivel superior establecen su contribución para vincularse adecuadamente a las necesidades de la sociedad a través de la generación de ciencia, tecnología e investigación. En ese sentido, la situación social, política y económica en la que se desenvuelve la función del docente universitario, en concordancia con la propia cultura universitaria en cada entorno, influirán en gran medida sus percepciones e identidad para que el docente 
fortalezca su profesión (Caballero y Bolívar, 2015). Los países carentes de los aspectos mencionados anteriormente evidencian una educación de baja calidad y con ello difícilmente pueden sobresalir en un mundo globalizado y cada vez más competitivo. Asimismo, los cambios del entorno donde el profesorado universitario, generan transformaciones en sus funciones, tareas y roles que se les asignan (Mas Torelló, 2012). Dentro del marco de ideas, se percibe la necesidad de que el profesorado universitario sea desarrollado profesionalmente en las mejores condiciones a fin de cumplir adecuadamente las funciones que cumplen en las comunidades universitarias para el mejoramiento de la calidad educativa a nivel superior y coadyuvar con el desarrollo sostenible y sustentable de la sociedad.

La Universidad Central del Ecuador conforme el informe de evaluación emitido por el Consejo de Evaluación, Acreditación y Aseguramiento de la Calidad de la Educación Superior, está ubicada en la categoría "B" (Resolución No. 001-073-CEAACES-2013-292013); enfatizando, que en este proceso se consideraron metodologías de análisis multicriterio y el análisis de conglomerados, mediante cinco criterios: academia, eficiencia académica, investigación, organización e infraestructura. En este sentido, conviene acentuar que como subcriterio del criterio academia, está la calidad docente, que evalúa la experiencia en el ejercicio profesional, en producción científica/ académica; así como también, la formación académica del cuerpo docente de la carrera (CEAACES, 2013). Dentro de este marco, resulta indispensable comprender el papel que juegan los procesos de desarrollo profesional del profesorado universitario. Precisamente, el análisis de los datos que preceden, motivo a indagar mediante las narrativas del profesorado novel universitario, las dificultades que enfrentan en su función docente conforme sus vivencias. Recalcando, que en la institución objeto de estudio no existen estudios relacionadas con esta temática, por lo que la presente investigación, resulta de interés para los distintos actores de la comunidad universitaria.

Villacís (2014), señala "la Universidad ha tenido que enfrentar una escasez de profesores debido a la salida de unos 300 profesores en febrero de 2014. La edad, la jubilación complementaria y los requisitos académicos para poder dar clases son los factores que motivaron la masiva renuncia". Al respecto, se puede vislumbrar que, en forma progresiva, muchos docentes antiguos con gran trayectoria y experiencia profesional decidieron retirarse de la carrera profesional universitaria. Significa entonces, que esa gran experiencia profesional debe ser igualada o superada por los nuevos docentes para completar la planta docente conforme las necesidades institucionales. Consecuentemente, el profesorado principiante debe ser formado permanentemente para posibilitar su desarrollo profesional conforme las demandas de la sociedad.
El desarrollo profesional del profesorado novel universitario, es un proceso complejo, que ha pesar de existir algunas investigaciones, sigue planteando grandes retos a los distintos actores educativos (Castañeda y Adell, 2011). Asimismo, debe ser un proceso sistémico que permita mejorar la calidad educativa de las organizaciones, fortaleciendo distintas competencias profesionales con el propósito de incrementar el nivel de desempeño del profesorado acorde a las distintas funciones que cumplen en su entorno (Gonzálvez, Vicent, García, e Inglés, 2014). Por este motivo, en las comunidades universitarias, se deben facilitar procesos de formación profesional continuos para que los docentes nuevos y experimentados fortalezcan sus funciones básicas, como son: la docencia, la investigación y la gestión para vincular a la universidad con la comunidad (Díaz y del Toro, 2013). Por consiguiente, el desarrollo profesional comprende procesos de crecimiento, progreso, adecuación, en relación con las competencias profesionales requeridas para incrementar su desempeño profesional conforme las necesidades de desarrollo institucional y social del contexto (Antoli, 2016). Sobre la base de las ideas expuestas, el desarrollo profesional del profesorado universitario puede ser vislumbrado como una concepción compleja y polisémica que pretende englobar distintos significados a fin de encaminar mayores niveles de actuación profesional conforme nuevos desafíos y tendencias que requiere la educación superior acorde al nuevo milenio.

Por otro lado, el profesorado principiante requiere de una etapa de inducción y para ello demanda de procesos de formación profesional (Bozu, 2010). Dicho de otra manera, el profesorado novel puede ser comprendido como un profesional, que se inicia en sus actividades y posee poca experiencia en las funciones que debe cumplir (Bozu, 2009). Consecuentemente, el docente novel universitario, es considerado como un inexperto en los procesos institucionales (Lledó et al., 2015). Significa entonces, que el profesor novel, es una persona que se encuentra en un momento de transición entre los conocimientos, habilidades y actitudes aprendidas en sus procesos de formación académica, y la puesta en acción de lo aprendido en el marco real de la intervención pedagógica conforme los requerimientos de la comunidad universitaria, es decir, se caracteriza por que comienza a ejercer sus actividades laborales y posee escasa experiencia en la dinámica organizacional lo que justifica plenamente de procesos de formación y acompañamiento para desarrollarse profesionalmente.

El desarrollo profesional de los docentes noveles universitarios resulta ser un proceso complejo en las que pueden intervenir una serie de factores. Al respecto, Cardona (2008), asevera "buscamos constatar el pensamiento de los profesores acerca de aquellas medidas que es necesario adoptar para evitar, o paliar, las situaciones difíciles a las que ha de responder el docente en la escuela actual para lograr la calidad deseada" (p. 31). Por consiguiente, 
conviene valorar la preocupación por la formación de los profesores noveles, ya que resulta relevante para el sistema educativo universitario, porque en los primeros años de haberse incorporado a una institución educativa de nivel superior se forman y se consolidan la mayor parte de los conocimientos, habilidades y actitudes que utilizarán en el ejercicio de la profesión docente. Asimismo, (Nogueira, Cubillas, González, 2018), plantean "proceso de construcción y reconstrucción social, donde todos aprenden con alto grado de autonomía y creatividad, con las mejores vivencias y experiencias" (p. 183). Significa entonces que el desarrollo profesional demanda de un proceso complicado, donde intervienen varios factores, como: formación profesional, la recategorización, remuneraciones percibidas, entre otros aspectos. Por consiguiente, conviene aplicar distintos modelos de desarrollo profesional que han logrado buenos resultados e invertir para la permanencia del profesorado en la universidad y asegurar que la enseñanza superior sea de calidad con el profesorado formado profesionalmente en las mejores condiciones.

Por su parte, (Cortés, Leite y Rivas, 2014) defienden "una perspectiva narrativa nos puede ofrecer la posibilidad, como se ha puesto de manifiesto en esta experiencia, de integrar estos elementos de discusión y análisis como base de la comprensión y reconstrucción del significado de la práctica educativa y/o docente" (p. 212). Evidentemente entonces, se puede dilucidar que resulta fundamental recurrir a los relatos de las vivencias de los participantes, como instrumentos de investigación que permiten visualizar e interpretar cómo se construye la idea del desarrollo profesional de un colectivo expuesto sistemáticamente a cambios. Es fundamental recalcar que una perspectiva narrativa puede ofrecer la posibilidad de integrar elementos de discusión y análisis como base de la comprensión y reconstrucción del significado de la práctica educativa y/o docente. A fin de cuentas, comprender la construcción de la identidad docente, es una forma de repensar nuevas vías de desarrollo profesional, desde su experiencia en las propias aulas.

Precisamente, investigaciones y trabajos como el de Dunkin (citado en Bozu, 2009) sostiene "uno de los pocos estudiosos de este tema, los problemas que padecen los profesores universitarios principiantes están fundamentalmente referidos a docencia, recursos, investigación y a los compañeros" ( $p$. 324). En esta perspectiva, se puede determinar que las dificultades que se manifiestan en el desarrollo profesional del profesorado novel tienen relación con las funciones básicas que cumple con su gestión en la comunidad universitaria.

El objetivo de estudio se encaminó para establecer las problemáticas del profesorado novel universitario en su función docente, desde sus propias narrativas. Al ser una investigación con enfoque cualitativo, se desarrolló una pregunta directriz para su comprensión y significación, más que para la verificación o refutación de una hipótesis. Significa entonces, que la cuestión de investigación se constituyó en el eje transversal de la investigación y se la formuló, de la siguiente manera:

¿Qué problemáticas existen en la función docente desde las narrativas del profesorado novel de la Universidad Central del Ecuador durante el año 2014?

\section{MÉTODOS}

\section{Población de estudio}

Se seleccionaron 68 profesores que empezaban sus funciones como docentes en la Universidad Central del Ecuador. La distribución por género fue de 37 hombres y 31 mujeres. En ambos grupos la participación fue voluntaria, brindaron su consentimiento para que se grabarán sus entrevistas. Los participantes fueron saludables y fluctuaban en edades desde 25 años hasta 45 años mayoritariamente.

\section{Metodología}

Se realizó un estudio investigativo con enfoque cualitativo con el criterio de comprender e interpretar el significado de las narrativas de los protagonistas. En este sentido, se aplicaron tres fases fundamentales: En primer lugar, la fase preparatoria o inicial, que contempló dos etapas esenciales: la reflexión teórica referencial y el diseño del trabajo en el campo. En segundo lugar, la fase de desarrollo, donde se aplicaron tres etapas: acceso al campo, recopilación de datos y análisis de los datos. En tercer lugar, la fase del informe de presentación de resultados para su difusión en la comunidad científica.

La institución objeto de estudio fue la Universidad Central del Ecuador, se planteó una cuestión específica sobre las problemáticas que cumple en su función docente con la intención de provocar respuestas de los protagonistas desde sus vivencias en busca de obtener una perspectiva general del problema investigado, el proceso de recopilación de la información fue desarrollado durante los meses de julio y agosto del 2014 como un componente esencial de la tesis doctoral del autor en investigación educativa desarrollado mediante convenio específico de colaboración académica y científica internacional en el área de educación entre la Universidad de Alicante de España y la Universidad Central del Ecuador suscrita el 28 de noviembre de 2013.

El muestreo fue no probabilístico, de tipo intencional, dirigida a 68 profesores nuevos provenientes de 17 Facultades, distribuidas en 4 áreas del conocimiento: Humanidades, Ciencias de la Vida, Ciencias Sociales, Físico Matemática con la finalidad de que la muestra sea representativa en función de las características de la realidad institucional conforme a su estructura y dinámica organizacional. Con relación a los datos sociodemográficos de la muestra, la mayoría tenía hasta 4 años de experiencia laboral en la institución (86,71\%), laborando a tiempo completo $(82,35 \%)$ y ubicados en la categoría Auxiliar (86,76\%). 
La técnica empleada para la recopilación de información, fue la entrevista mediante una guía estructurada que permitió profundizar sobre la cuestión investigada, fue validada mediante la triangulación de expertos en investigación cualitativa.

Para el proceso de tratamiento y análisis de los datos cualitativos fue necesario recurrir al software informático Aquad 6, Huber y Gürtler (citado en Fombona, Iglesias y Lozano, 2016) herramienta que facilita la interpretación de las narrativas de los participantes en una serie de temáticas emergentes. Para lo cual, preliminarmente se elaboró una matriz de coherencia (objetivos, cuestiones de investigación, marco conceptual, preguntas de entrevista, categorías, códigos y subcódigos) a partir de las unidades de texto de las narrativas de los entrevistados. Enfatizando, que dicha matriz fue validada mediante la triangulación de investigadores expertos, lo que permitió el diseño conceptual y descripción de las temáticas a través de un sistema de códigos inferenciales generados para su interpretación.

\section{RESULTADOS}

Sobre las problemáticas en la función docente, se obtuvieron los siguientes hallazgos:

Como se observa en la figura 1 en el subcódigo 1.1 .1 , se aprecia que sobresale un $26,01 \%$ del total de los entrevistados, quienes afirman que los problemas en la docencia de sus asignaturas, se deben a que los estudiantes no dominan conocimientos básicos sobre los contenidos curriculares de las asignaturas que

Tabla 1. Frecuencias absolutas y porcentajes de las problemáticas en la función docente

\begin{tabular}{|c|c|c|c|}
\hline \multicolumn{4}{|c|}{$\begin{array}{l}\text { CATEGORÍA } \\
\text { 1. Problemáticas en la investigación }\end{array}$} \\
\hline CÓDIGOS & SUBCÓDIGOS & FA & $\%$ \\
\hline \multirow{5}{*}{$\begin{array}{l}2.1 \text { Problemáticas de condicionantes } \\
\text { personales del participante }\end{array}$} & 2.1.1 Falta de formación & 35 & 21.74 \\
\hline & 2.1.2 Poco dominio de TICs & 2 & 1.24 \\
\hline & 2.1.3 Poca experiencia & 4 & 2.48 \\
\hline & 2.1.4 Reducido tiempo & 6 & 3.73 \\
\hline & TOTAL & 47 & 29.19 \\
\hline \multirow{6}{*}{$\begin{array}{l}2.2 \text { Problemáticas de condicionantes } \\
\text { institucionales }\end{array}$} & 2.2.1 Gestión inadecuada & 37 & 22.98 \\
\hline & 2.2.2 Discriminación & 5 & 3.11 \\
\hline & 2.2.3 Soporte tecnológico deficiente & 2 & 1.24 \\
\hline & 2.2.4 Recarga de actividades & 7 & 4.35 \\
\hline & 2.2.5 Baja Cultura investigativa & 55 & 34.16 \\
\hline & TOTAL & 106 & 65.84 \\
\hline \multirow{2}{*}{ 2.3 No detectan condicionantes } & 2.3.1 No detectan condicionantes & 8 & 4.97 \\
\hline & TOTAL & 8 & 4.97 \\
\hline & TOTAL (FA) y (T \%) & 161 & 100.00 \\
\hline
\end{tabular}

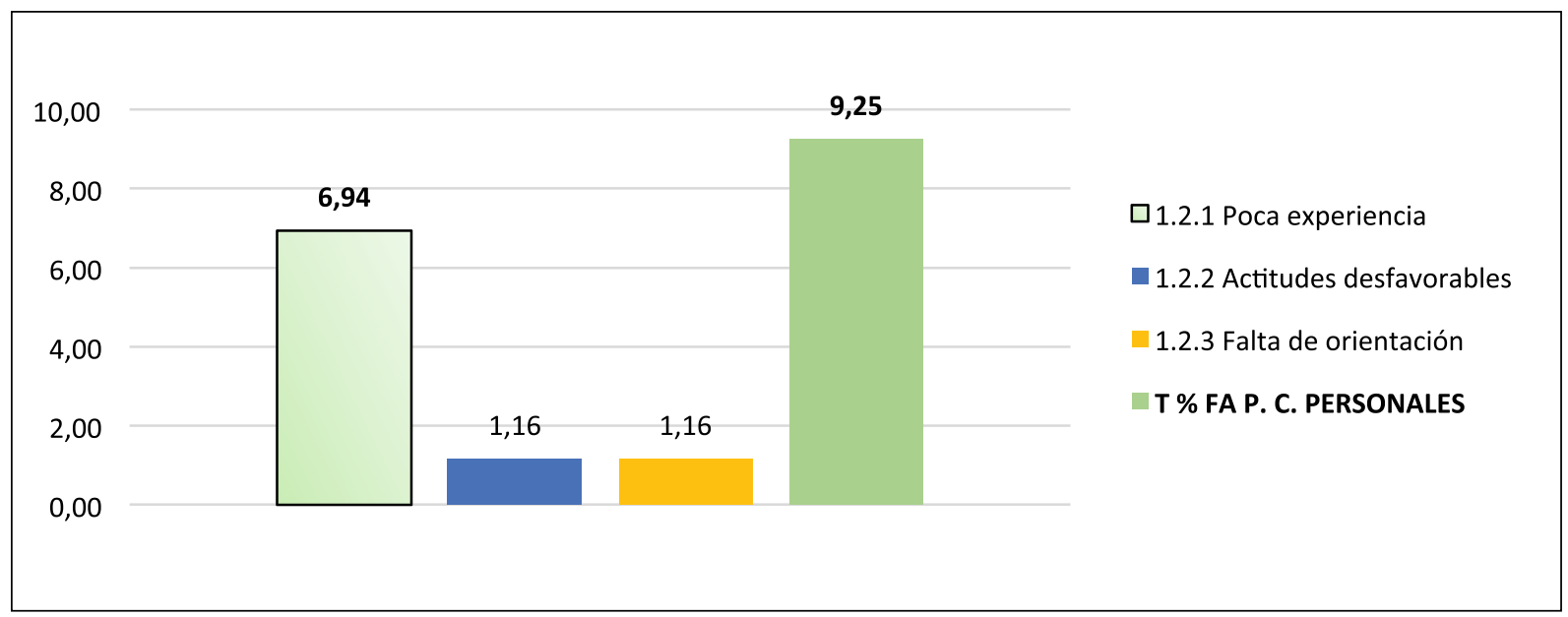

Figura 1. Porcentajes de las problemáticas que se deben a condicionantes estudiantiles. 
cursan y dificultan su labor docente. Las siguientes narrativas confirman dichas aseveraciones:

En la docencia, el nivel básicamente. Es el nivel académico con el que vienen los alumnos. Yo manejo cursos de niveles inferiores. Entonces de los colegios no vienen con los conocimientos suficientes (Participante 024).

En la docencia se me han presentado dificultades cognitivas. Es decir, los estudiantes tienen un ritmo de aprendizaje bastante lento. Son bastantes reacios a las cosas nuevas. No tienen hábitos de estudio. En definitiva, he tenido problemas con el aprendizaje de los estudiantes (Participante 004).

Conforme la figura 2 , se puede visualizar que en el subcódigo 1.2.1 un 6,94\% del total de los participantes quienes aseveran que los problemas en la docencia de sus asignaturas, se deben a su poca experiencia en asignaturas nuevas que les fueron asignadas por sus autoridades, en razón, de que resultan complejas por su poco dominio. Los siguientes fragmentos, dan muestra de ello:

A veces la falta de un poquito de experiencia, la falta de cancha, porque muchas veces no podemos tener a la mano la experiencia para uno poder expresarse bien y para llegar a cierto punto (Participante 012).

Yo pienso que la principal es que me he incorporado en la universidad como docente después de aproximadamente catorce años de laborar en otras actividades (Participante 041).

La figura 3, permite observar que en el subcódigo 1.3.1 se detalla que un $38,15 \%$ del total de los protagonistas afirman que los problemas en la docencia de sus asignaturas, se deben a la falta de apoyo de sus autoridades frente a diferentes requerimientos, como son: infraestructura para aulas y laboratorios, limitaciones en la programación de actividades de sus autoridades, haciendo sentir su malestar respecto a la gestión de sus autoridades considerada como deficiente para el desarrollo de la unidad académica donde laboran. Las siguientes narrativas, demuestran tales aseveraciones:

La falta de apoyo que hay de las autoridades раra poder equipar los laboratorios con equipos adecuados de acuerdo a la carrera (Participante 019).

Yo pienso que aquí en la carrera no ha existido un proceso de seguimiento a una programación. Cada uno de los docentes que venían a trabajar en la carrera daba su materia, su cátedra, en dependencia de su experiencia y de lo que pensaban que era lo correcto. En cierta forma, no existía realmente, repito, una programación de las asignaturas (Participante 008).

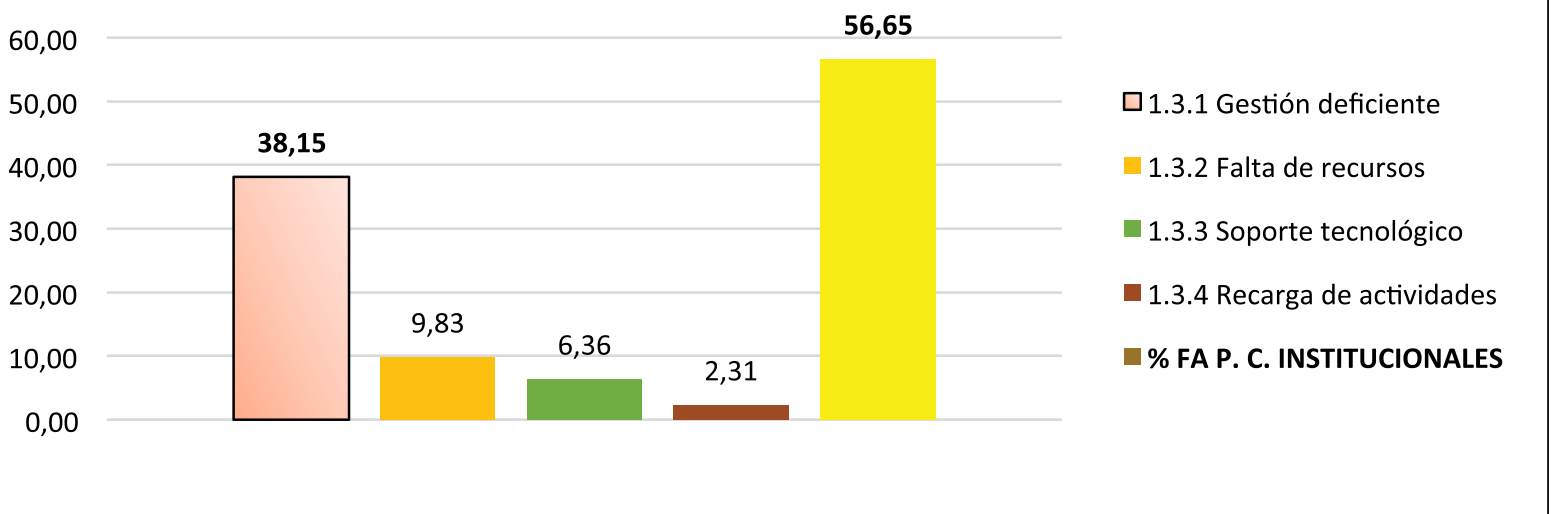

Figura 2. Porcentajes de las problemáticas que se deben a condicionantes personales.

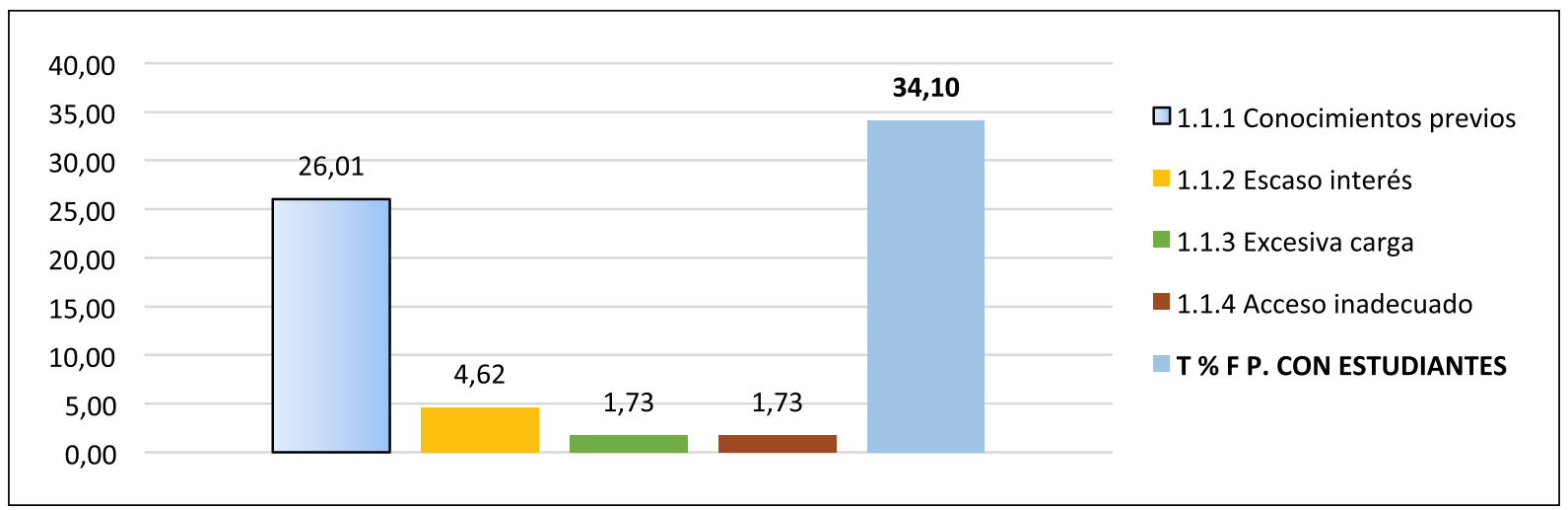

Figura 3. Porcentajes de las problemáticas que se deben a condicionantes institucionales. 
Los principales problemas que se tiene referente a la infraestructura de las aulas, la falta de computadores, de proyectores, la falta de textos actualizados, principalmente eso en cuanto a infraestructura (Participante 042).

Tenemos un sistema de Universidad muy burocrática, digamos que se complica en procesos de adquisición de equipamiento, reactivos para laboratorios y también de pronto, el descuido de las autoridades (Participante 052).

\section{DISCUSIÓN}

Los resultados obtenidos en el presente estudio, permiten descubrir que un $56,65 \%$ de participantes afirma las condicionantes institucionales como la causa principal que repercute en la problemática de su función docente. En este sentido, las causas que más han reiterado los protagonistas y muestran sus dificultades, han sido: i) gestión deficiente de las autoridades; ii) falta de recursos para los procesos académicos; iii) deficiente soporte tecnológico para acceso a internet y falta de mantenimiento de equipos; iv) recarga de actividades emanadas por sus autoridades, de donde sobresale un 38,15\% de noveles que aseveran en sus narrativas falta de apoyo de las autoridades para atender sus requerimientos de los laboratorios y de las aulas, evidenciadas en sus narrativas: por la falta de proyectores, falta de textos actualizados y situaciones burocráticas en la gestión administrativa que afectan su labor docente y que no permiten su participación para resolver las problemáticas. Consecuentemente, se puede desprender que no existe una mayoría en la diversidad de los docentes participantes, como para juzgar que la gestión de las autoridades sea deficiente. No obstante, es importante considerar que la falta de apoyo de las autoridades, se constituye en un factor clave el cual repercute en las problemáticas de las funciones docentes del profesorado novel.

Evidentemente los hallazgos de la presente investigación guardan relación con el estudio de Mas Torelló (2011), donde se afirma que el profesor universitario no tiene una adecuada preparación psicopedagógica que, sumada a la falta de apoyo, puede ser peligroso para los estudiantes y para el desarrollo profesional del profesorado. Ello es concordante con lo que en este estudio se devela. Ante estas aseveraciones, conviene enfatizar que los docentes noveles universitarios experimentan diferentes problemas en su función docente por la poca experiencia que tienen en sus funciones, de conformidad a la estructura y dinámica organizacional. En este sentido, se evidencia que la falta de apoyo por parte de las autoridades interfiere en el desarrollo de sus actividades docentes. Por consiguiente, resulta fundamental resaltar que el desarrollo profesional del profesorado novel universitario debe ser considerado por parte de las autoridades institucionales, como un eje transversal el cual contribuirá al mejoramiento del desempeño docente e incrementará la calidad educativa que se oferta en la universidad.

Igualmente, los resultados conseguidos concuerdan con el estudio de Eirín, García y Montero (2009), donde se defiende que debería vislumbrarse un rol más activo de los profesores en el diseño y la implementación de iniciativas orientadas a su desarrollo profesional. Ello está relacionado con lo que en este estudio se devela. Es evidente entonces, que resulta esencial la participación del docente en la previsión de actividades enfocadas al desarrollo profesional del profesorado universitario. En este sentido, conviene destacar que en las comunidades universitarias es fundamental investigar mediante un diagnóstico situacional, qué tipo de necesidades y expectativas tienen los docentes universitarios en las distintas unidades académicas para poder comprender e interpretar sus necesidades acordes a las funciones que cumplen. Esta situación conlleva a la necesidad de implementar programas y proyectos que fortalezcan el desarrollo profesional del profesorado novel universitario.

Por otro lado, según los hallazgos derivados del presente estudio, se pudo evidenciar que un 34,10\% del profesorado novel universitario entrevistado, asegura, como otra causa que trasciende en los problemas de su función docente en sus asignaturas se deben a condicionantes del estudiantado, dejando entrever que sus opiniones responden a: i) falta de conocimientos previos; ii) el escaso interés hacia los estudios; iii) excesiva carga de actividades académicas; iv) el acceso inadecuado de los estudiantes a las unidades académicas, de donde prevalece un 26,01\% de entrevistados los cuales en sus relatos, aseguran que sus problemas en la tarea docente se deben a que sus estudiantes no dominan contenidos mínimos obligatorios de las asignaturas que cursan y sus condiciones demandan de la aplicación de otro tipo de metodologías.

En forma similar, estos resultados coinciden con los estudios (Antón y Pérez, 2015; Madero, Fondón y Sarmiento, 2010), ellos sostienen que es fundamental atender los problemas relacionados con el comportamiento y actitud de los estudiantes en clase y la necesidad de mejorar el modelo didáctico personal del docente novel. Estas evidencias son semejantes con lo que en este estudio se revela. Por consiguiente, se puede destacar, que resulta fundamental la necesidad de mejorar los procesos metodológicos del profesorado novel universitario раra atender situaciones académicas a nivel de aula, diferenciando estilos y ritmos de aprendizaje, exaltando la atención que se debe dedicar al fortalecimiento del modelo didáctico personal del docente novel con el objetivo de garantizar su intervención en los procesos académicos. En esta perspectiva, se justifica la necesidad de un proceso de formación profesional continuo para el profesorado novel universitario a fin de incrementar su nivel de desempeño en su labor docente.

En cambio, conforme los hallazgos, un 9,25\% de 
los docentes noveles universitarios participantes asevera que sus problemáticas en la función docente se deben a condicionantes personales las cuales se evidencian por diferentes causales, que responden a: i) poca experiencia en la labor docente; ii) actitudes desfavorables en el ambiente laboral; iii) falta de orientación o malas vías de comunicación; de donde predomina, un $6,94 \%$ de participantes quienes afirman en sus relatos que su principal problema es la poca experiencia en su labor docente respecto a nuevas cátedras que les fueron asignadas para impartir sus clases.

Estos resultados logrados en la presente investigación, son afines al estudio de Bozu (2009) quien asegura, que el profesor novel se caracteriza por tener poca o ninguna experiencia en la labor docente. Significa entonces, que se debe exaltar, el profesorado novel en la comunidad universitaria, no reúne las suficientes competencias profesionales para desempeñar adecuadamente sus actividades pedagógicas. Sin embargo, si a esto se agrega que el docente principiante no se desenvuelve en un ambiente idóneo para su ejercicio profesional, su nivel de desempeño en la labor docente, no va ser la más adecuada.

Pretendiendo dar un significado a los resultados obtenidos en la presente investigación, desde el punto de vista de los protagonistas respecto a la cuestión investigada, se podría interpretar que los docentes noveles participantes, han destacado que existe falta de apoyo de sus autoridades para atender diferentes requerimientos necesarios en sus procesos académicos, lo que indica una tendencia de insatisfacción respecto a la gestión de las autoridades para la resolución de problemas en su función docente.

Conocidos los resultados del presente estudio, conviene prestar atención a los estudios (Ávalos, 2009; Gómez, 2010; Lledó et al., 2015; Mayor Ruiz y Sánchez Moreno, 2006) quienes acentúan que el desarrollo profesional del novel es fundamental para su identidad universitaria, ya que los sistemas educativos deben asumir el reto de la calidad. Estas afirmaciones, permiten destacar la importancia que tienen los profesores universitarios, los cuales deben ser competentes para la eficacia de la educación superior. Dentro de este marco, se puede acentuar que el profesorado nuevo universitario, tiene que estar preparado en las mejores condiciones para cumplir sus funciones docentes las cuales deben caracterizarse por responder a los grandes desafíos que enfrenta la educación superior en el nuevo milenio. Consecuentemente, el desarrollo profesional del profesorado novel universitario, debe estar empeñado constantemente hacia la excelencia académica. En este sentido, conviene reflexionar que el profesor novel es una persona nueva, que se encuentra en un proceso de transición y de puesta en acción de lo aprendido conforme su experiencia preliminar en la comunidad universitaria.

Con la finalidad de comprender el significado de las opiniones del profesorado participante, se puede indicar que los participantes en sus relatos deslindan su responsabilidad en sus funciones docentes, enfatizando que sus dificultades responden a condicionantes institucionales y estudiantiles. Sin embargo, se puede apreciar que no consideran en sus narrativas, otros elementos que son fundamentales para su actividad docente, como es el dominio del tratamiento curricular que intervienen en los procesos de enseñanza - aprendizaje, es decir, el profesorado novel, no enfoca sus respuestas respecto al componente curricular que contempla: los objetivos, contenidos, secuenciación, tratamiento de contenidos, metodología, recursos y evaluación, que debe ser dominado por el profesorado para su intervención pedagógica en el acto educativo y ser concordante con un determinado modelo pedagógico que responda a un tipo de ser humano que demanda la sociedad. Significa entonces, que sus respuestas pueden estar basadas en ciertos criterios e indicadores los cuales han sido considerados por el Consejo de Evaluación, Acreditación y Aseguramiento de la Calidad de Educación Superior que actualmente se están empleando en las evaluaciones de las distintas unidades académicas de las comunidades universitarias en el contexto ecuatoriano, y han permitido comprobar debilidades en situaciones de infraestructura y recursos que son necesarios para el proceso de aprendizaje.

Desde el punto de vista del autor, resulta imperante reflexionar sobre el hecho de que el profesorado novel universitario en el inicio de sus funciones interactúa con estudiantes con diversas expectativas, se relaciona con docentes más experimentados, enfrenta cambios de normativas establecidas para los escenarios educativos. Estas situaciones, representan grandes desafíos y retos que pueden motivar al profesorado principiante, a contribuir con el mejoramiento de la calidad educativa. No obstante, al sentir que no recibe apoyo de sus autoridades y de sus compañeros pueden repercutir desfavorablemente en su labor docente y su aspecto emocional. Consecuentemente, para profundizar en las problemáticas del profesorado novel universitario convendría indagar en futuras investigaciones si se imparten cursos de inducción a los docentes principiantes, si existen programas de actualización sobre procesos metodológicos, si existen talleres de apoyo emocional mediante tutorías, y si se brindan facilidades para la creación de obras didácticas, entre otros factores que pueden afectar la labor docente.

\section{CONCLUSIONES}

En definitiva, los resultados obtenidos en este estudio supondrían una importante contribución al campo del desarrollo profesional del profesorado universitario, porque se han develado tres factores esenciales que intervienen en las problemáticas de las funciones docentes del profesorado novel universitario: En primer lugar, la falta de apoyo de las autoridades para atender sus requerimientos 
a nivel de aula y de laboratorios, que son necesarios para el fortalecimiento de sus procesos académicos. En segundo lugar, limitaciones en el dominio de conocimientos básicos de los estudiantes en asignaturas que cursan e interfieren desfavorablemente en los procesos didácticos. En tercer lugar, se evidencia que se constituye en un problema relevante, la poca experiencia que tienen en sus tareas docentes cuando tienen que impartir asignaturas nuevas conforme las necesidades institucionales y que les resultan complejas para el proceso de enseñanza.

\section{RECOMENDACIONES}

- En la comunidad universitaria investigada, se deben mejorar los procesos de formación profesional del profesorado novel universitario aplicando distintos modelos de formación profesional.

- Es imprescindible seguir emprendiendo investigaciones con enfoques cualitativos y cuantitativos para develar diversos factores que influyen en la función docente del profesorado novel y que pueden perjudicar su desarrollo profesional.

- Es fundamental, diferenciar distintas fases y procesos que contribuyan al desarrollo profesional del profesorado universitario a fin de perfeccionar las competencias profesionales de los docentes noveles y experimentados.

- Es indispensable, el mejoramiento de la gestión institucional mediante sistemas de gestión innovadores para consolidar la calidad educativa en las instituciones educativas de nivel superior.

\section{REFERENCIAS}

- Antoli, V. (2016). Desarrollo profesional del profesor universitario. Campo Abierto. Revista de Educación, 10 (1), 175-197. Recuperado de http:// mascvuex.unex.es/revistas/index.php/campoabierto/article/view/2622

- Antón, D., y Pérez, M. (2015). La docencia universitaria novel como mejora metodológica. Opción, 31 (3), 104-124. Recuperado de http://200.74.222.178/index.php/opcion/article/ viewFile/20478/20389

- Ávalos, B. (2009). La inserción profesional de los docentes. Profesorado: Revista de curriculum y formación del profesorado, 13(1), 44-59. Recuperado de http://www.ugr.es/ recfpro/rev131ART3. pdf

- Bozu, Z. (2009). El profesorado universitario novel y su proceso de inducción profesional. Magis. Revista Internacional de Investigación en Educación, 1 (2), 317-328. Recuperado de http://revistas.javeriana.edu.co/index.php/MAGIS/article/ view/3387/2576

- Bozu, Z. (2010). El profesorado universitario novel: estudio teórico de su proceso de inducción o socialización profesional. Revista Electrónica de Investigación y Docencia (REID), (3), 55-72. Recuperado de http://dialnet.unirioja.es/servlet/articulo?codigo=3186125
- Caballero, K., y Bolívar, A. (2015). El profesorado universitario como docente: hacia una identidad profesional que integre docencia e investigación. REDU: Revista de Docencia Universitaria, 13(1), 57-77. Consultado el 3 de marzo de 2014. Recuperado de http://dialnet.unirioja.es/servlet/articulo?codigo $=5027836$

- Cardona Andújar, José (2008). Problemática actual del profesorado: algunas soluciones. Enseñanza: Revista interuniversitaria de didáctica, (26), 29-56. Recuperado de http://campus.usal. es/ revistas_trabajo/index.php/0212-5374/article/viewFile/684/858

- Castañeda, L., y Adell, J. (2011). El desarrollo profesional de los docentes en entornos personales de aprendizaje (PLE). En Roig Vila, R. y Laneve, C. (Eds.) La práctica educativa en la Sociedad de la Información: Innovación a través de la Investigación / La práctica educativa nella Societa dellinformazione: $L$ innovazione attraverso la ricerca. Alcoy: Marfil. 83 - 95. Recuperado de https:// digitum.um.es/xmlui/bitstream/10201/24647/1/ CastanedaAdell2011preprint.pdf

- CEAACES(2013). Resolución No. 001-073-CEAACES-2013-29. El Consejo de Evaluación, Acreditación y Aseguramiento de la Calidad de la Educación Superior. Consultado el 19 de febrero de 2018. Recuperado de http://www.ceaaces.gob. ec/sitio/wp-content/uploads/2013/10/RESOLUCIO\%CC\%81N-No.-001-073-CEAACES-201329-UCE.pdf

- CEAACES (2013). Informe General sobre la Evaluación, Acreditación y Categorización de las Universidades y Escuelas Politécnicas. Dirección de Acreditación de Universidades y Escuelas Politécnicas. Recuperado de https://universidadsociedadec.files.wordpress.com/2014/04/ceaaces-informe-general-eval-accred-categn.pdf

- Cortés González, P., Leite Méndez, A., y Rivas Flores, (2014). Un enfoque narrativo de la identidad profesional en profesorado novel. pp. 199-214. Tendencias Pedagógicas, (24), 199-214. Recuperado de https://revistas.uam.es/tendenciaspedagogicas/article/download/2101/2199

- Díaz, R. T., y del Toro, P. S. (2013). Estrategias de intervención para la formación de competencias profesionales en la educación superior. Pedagogía Universitaria, 15 (5), 39-53. Recuperado de http:// cvi.mes.edu.cu/peduniv/index.php/peduniv/article/view/554/553

- Eirín Nemiña, R., García Ruso, H. M., y Montero Mesa, L. (2009). Desarrollo profesional y profesionalización docente. Perspectivas y problemas. Profesorado Revista de currículum y formación del profesorado. 13 (2), 1-13. Recuperado de http://digibug.ugr.es/bitstream/10481/7378/1/ rev132COL3.pdf

- Fombona, J., Iglesias, M., y Lozano, I. (2016). El trabajo colaborativo en la educación superior: una competencia profesional para los futuros docentes. Educação \& Sociedade, 37(135), 
519-538. https://dx.doi.org/10.1590/ES010173302016147914

- Gómez, A. (2010). Nuevas exigencias y escenarios para la profesión docente en la era de la información y de la incertidumbre. Revista Interuniversitaria de Formación del Profesorado, (68) 24, 17-36. Recuperado de http://dialnet.unirioja.es/servlet/ articulo?codigo $=3276040$

- Gonzálvez M., Vicent M., García, J., e Inglés C. (2014). La concepción del desarrollo profesional docente como factor influyente en la actividad educativa. XII Jornadas de Redes de investigación en Docencia Universitaria. El reconocimiento docente: innovar e investigar con criterios de calidad, Universidad de Alicante, España, 650-665. Recuperado de http://rua.ua.es/dspace/bitstream/10045/41831/1/2014_XII_Jornadas_Redes_51.pdf

- Lledó Carreres, A., Gonzálvez Macià, C., Vicent Juan, M., Hernández Amorós, M. J., Delgado Domenech, B., Lorenzo Lledó, G., ... y Martínez Monteagudo, M. C. (2015). Profesorado novel y tutoría entre iguales en innovación tutorial. XIII Jornadas de Redes de investigación en Docencia Universitaria. Nuevas estrategias organizativas y metodológicas e la formación universitaria para responder a la necesidad de adaptación y cambio. Universidad de Alicante. España, 668-684. Recuperado de http://rua.ua.es/dspace/bitstream/10045/49159/1/XIII_Jornadas Redes 51. pdf

- Madero Ayora, M. J., Fondón García, I., y Sarmiento Vega, M. A. (2010). Principales problemas de los profesores principiantes en la enseñanza universitaria. Formación Universitaria. 3 (2), 2128. Recuperado de https://idus.us.es/xmlui/bitstream/handle/11441/17057/file_1.pdf?sequen- ce $=1$ \&isAllowed $=y$

- Mas Torelló, Ó. (2011). El profesor universitario: sus competencias y formación. Profesorado: Revista de curriculum y formación del profesorado. 15 (3), 196-211. Recuperado de http://ddd.uab. cat/pub/artpub/2011/112747/profesorado_ a2011v15n3col1.pdf

- Mas Torelló, Ó. (2012). Las competencias del docente universitario: la percepción del alumno, de los expertos y del propio protagonista. REDU. Revista de Docencia Universitaria, 10 (2), 299-318. Recuperado de http://red-u.net/redu/index.php/ REDU/article/view/381

- Mayor Ruiz, C., y Sánchez Moreno, M. (2006). Los jóvenes profesores universitarios y su formación pedagógica: claves y controversias. Revista de Educación, (339), 923-946. Recuperado de http:// www.revistaeducacion.mec.es/re339/re339a40. pdf

- Nogueira, G. A., Cubillas-Quintana, F., y González-Fernández, Z. (2018, julio-octubre). Reflexiones teóricas sobre la preparación del docente para la orientación profesional en Angola. Pedagogía y Sociedad, 21(52), 162-186.Recuperado de http:// revistas.uniss.edu.cu/index.php/pedagogia-y-sociedad/article/view/691

- Villacís David (2014, junio 24). La Universidad Central busca otro perfil de docentes frente a las renuncias. El Comercio. Recuperado de http:// www.elcomercio.com/tendencias/universidad-central-busca-perfil-docentes-frente-renuncias-profesores-escacez.html 